\title{
Creating a (Quantum?) Constraint, in Pre Planckian Space-Time Early Universe via the Einstein Cosmological Constant in a One to One and Onto Comparison between Two Action Integrals
}

\author{
Andrew Walcott Beckwith \\ Physics Department, College of Physics, Chongqing University Huxi Campus, Chongqing, China \\ Email: Rwill9955b@gmail.com, abeckwith@uh.edu
}

How to cite this paper: Beckwith, A.W. (2017) Creating a (Quantum?) Constraint, in Pre Planckian Space-Time Early Universe via the Einstein Cosmological Constant in a One to One and Onto Comparison between Two Action Integrals. Journal of High Energy Physics, Gravitation and Cosmology, 3, 167-172.

https://doi.org/10.4236/jhepgc.2017.32017

Received: December 28, 2016 Accepted: February 26, 2017

Published: March 1, 2017

Copyright $\odot 2017$ by author and Scientific Research Publishing Inc. This work is licensed under the Creative Commons Attribution International License (CC BY 4.0).

http://creativecommons.org/licenses/by/4.0/

\begin{abstract}
We are looking at comparison of two action integrals and we identify the Lagrangian multiplier as setting up a constraint equation (on cosmological expansion). Two action integrals, one which is connected with quantum gravity is called equivalent to another action integral, and the $2^{\text {nd }}$ action integral has a Lagrangian multiplier in it. Using the idea of a Lagrangian multiplier as a constraint equation, we draw our conclusions in a 1 to 1 and onto assumed equivalence between the two action integrals. The viability of the 1 to 1 and onto linkage between the two action integrals is open to question, but if this procedure is legitimate, the conclusions so assumed are fundamentally important.
\end{abstract}

Keywords

Ricci Scalar, Inflaton Physics

\section{Basic Idea, Can Two First Integrals Give Equivalent Information?}

Our supposition is that if we wish to make an equivalence between two action integrals, i.e., first integrals that we need to have a 1 to 1 and onto linkage between the integrands, in the two cases so referenced.

To do this, we are making several assumptions.

1) The two mentioned integrals are evaluated from a Pre Planckian to Planckian space-time domain, i.e. in the same specified integral of space-time. 
2) In the process of doing so, the Universe is assumed to avoid the so called cosmic singularity. In doing so, assuming a finite "Pre Planckian to Planckian" regime of space time is similar to that given in [1] [2].

3) The integrands in the two integrals are assumed to have a 1-1 and onto relationship to one another. We will be identifying the components of the two integrands which are assumed to be proportional to each other. This idea is the foundation of our approach. The two references [1] [2] have in their own formulation specific Lagrangian formulations and a criticism our approach is that the references we are using for first integrals, namely [3] [4] are not giving action integrals identical as to [1] [2]. Our answer is that we reference [1] [2] specifically as to how to avoid the Penrose singularity theorem [5], and that not enough is known as to rule out the nonsingular starting point of the universe as having the same content for Lagrangians as given in [3] [4]. i.e., for Pre Planckian space time, so long as [5] is avoided, presumably our three assumptions for comparison can be made, so long as we adhere to the "path integral" idea as represented by [6] as equivalent to what is stated in [1] [2].

\section{Specifying the Particulars of the Two First Integrals in Pre-Planckian to Planckian Space-Time}

Before proceeding, it is advisable to define some of the symbols which will be used in the integrals and the integrands in our document.

First of all, we have what is known as a scale factor $a(t)$. Which is nearly zero, in the Pre Planckian regime of space-time if we assume [5] does not hold, and that $a(t)$ is 1 in the present era. A good reference as to the physics behind how we set up $a(t)$ is [7]. In addition we will define, for the purpose of analysis, of the integrals, the following symbols as given in [1], for the Quantum paths sensitive first integral, with

$$
\begin{aligned}
& \int \mathrm{d} t \sqrt{g_{t t}} V_{3}(t)=V_{4}(t) \sim 8 \pi^{2} r^{4} / 3 \\
& \& V_{3}(t)=2 \pi^{2} a(t)^{3} / 3 \\
& \& k_{2}=9\left(2 \pi^{2}\right)^{2 / 3}
\end{aligned}
$$

These are the purported volume elements of the [3] first integral. The second first integral is using the usual $G R$ inputs as defined by Padmanbhan in [4]. To review what is meant by first integrals we refer the readers to [6] [8] [9] [10].

Roughly put, according to [8] [9] [10] a Lagrangian multiplier invokes a constraint of how a "minimal surface" is obtained by constraining a physical process so as to use the idea of [8] [9] [10] which invokes the idea of minimization of a physical processes. In the case of [3], the minimization process is implicitly that, if $a(t)$ were a scale factor as defined by Roos, [7] and if $g_{t t}$ were a time component of a metric tensor, which we will later define via [11] [12].

Here, the subscripts 3 and 4 in the volume refer to 3 and 4 dimensional spatial dimensions, and this will lead to us writing, via [3] a $1^{\text {st }}$ integral as defined by [3] [3], in the form, if $G$ is the gravitational constant, that if we have following [3], a 
first integral defined by

$$
S_{1}=\frac{1}{24 \pi G} \cdot\left(\int \mathrm{d} t \sqrt{g_{t t}}\left(\frac{g_{t t} \dot{V}_{3}^{2}}{V_{3}(t)}+k_{2} V_{3}^{1 / 3}(t)-\lambda V_{3}(t)\right)\right) .
$$

This should be compared against the Padmabhan $1^{\text {st }}$ integral [4] of the form, with the third entry of Equation (3) having a Ricci scalar defined via [13] and usually the curvature $\aleph$ set as extremely small, with the general relativity version of

$$
\begin{aligned}
& S_{2}=\frac{1}{2 \kappa} \int \sqrt{-g} \cdot \mathrm{d}^{4} x \cdot(\Re-2 \Lambda) \\
& \&-g=-\operatorname{det} g_{u v} \\
& \& \Re=6 \times\left(\frac{\ddot{a}}{a}+\left(\frac{\dot{a}}{a}\right)^{2}+\frac{\aleph}{a^{2}}\right) .
\end{aligned}
$$

Also, the variation of $\delta g_{t t} \approx a_{\min }^{2} \phi$ as given by [11] [12] will have an inflaton, $\phi$ given by [4]

$$
\begin{aligned}
& a \approx a_{\min } t^{\gamma} \\
& \Leftrightarrow \phi \approx \sqrt{\frac{\gamma}{4 \pi G}} \cdot \ln \left\{\sqrt{\frac{8 \pi G V_{0}}{\gamma \cdot(3 \gamma-1)}} \cdot t\right\} \\
& \Leftrightarrow V \approx V_{0} \cdot \exp \left\{-\sqrt{\frac{16 \pi G}{\gamma}} \cdot \phi(t)\right\} .
\end{aligned}
$$

Leading to [2]

$$
\phi \approx \sqrt{\frac{\gamma}{4 \pi G}} \cdot \ln \left\{\sqrt{\frac{8 \pi G V_{0}}{\gamma \cdot(3 \gamma-1)}} \cdot t\right\} .
$$

Here, we have that $a_{\min }$ is a minimum value of the scale factor presumably given by [2] as a tiny but non zero value. Or at least a quantum bounce as given by [1].

The innovation we will be looking at will be in comparing a 1-1 and onto equivalence, i.e. an information based isomorphism between $1^{\text {st }}$ integrals with a nod to [14]

$$
S_{1} \cong S_{2}
$$

We will be making a simple equivalence between the two first integrals via Equation (6) assuming that even in the Pre Planck-Planck regime that curvature $\aleph \quad$ will be a very small part of Ricci scalar $\mathfrak{R}$ and that to first approximation even in the Plank time regime, that to first order [13] has a value altered to be

$$
\mathfrak{R}=6 \times\left(\frac{\ddot{a}}{a}+\left(\frac{\dot{a}}{a}\right)^{2}+\frac{\aleph}{a^{2}}\right) \sim 6 \times\left(\frac{\ddot{a}}{a}+\left(\frac{\dot{a}}{a}\right)^{2}\right) .
$$

This last approximation will make a statement as to applying Equation (6) far easier may not be defensible, but we will use it for the time being. 


\subsection{Comparison between Equations ((2) and (3) with (5)-(7))}

In order to obtain maximum results, we will be stating that the following will be assumed to be equivalent.

$$
\sqrt{g_{t t}}\left(\frac{g_{t t} \dot{V}_{3}^{2}}{V_{3}(t)}+k_{2} V_{3}^{1 / 3}(t)-\lambda V_{3}(t)\right) \sim \frac{1}{2 \kappa} \int \sqrt{-g} \cdot \mathrm{d}^{3} x \cdot\left(6 \times\left(\frac{\ddot{a}}{a}+\left(\frac{\dot{a}}{a}\right)^{2}\right)-2 \Lambda\right)
$$

i.e.

$$
\sqrt{g_{t t}}\left(\frac{g_{t t} \dot{V}_{3}^{2}}{V_{3}(t)}+k_{2} V_{3}^{1 / 3}(t)\right) \sim \frac{1}{2 \kappa} \int \sqrt{-g} \cdot \mathrm{d}^{3} x \cdot\left(6 \times\left(\frac{\ddot{a}}{a}+\left(\frac{\dot{a}}{a}\right)^{2}\right)\right)
$$

And

$$
\sqrt{g_{t t}}\left(\lambda V_{3}(t)\right) \sim \frac{1}{2 \kappa} \int \sqrt{-g} \cdot \mathrm{d}^{3} x \cdot(2 \Lambda) .
$$

If the term $\Lambda$ is indeed a constant (i.e. we avoid Quinessence, and the vacuum energy is invariant), then Equation (10) puts a profound restriction upon $g_{t t}$ which will be elaborated upon in the next section. i.e. for the sake of Argument we will make the following assumptions which may be debatable, i.e.

$$
\sqrt{-g} \text { is approximately a constant. }
$$

For extremely small time intervals (in the boundary between Pre Planckian to Planckian physics boundary regime).

$$
g_{t t} \sim \delta g_{t t} \approx a_{\min }^{2} \phi
$$

The next section will be investigating the physical implications of such assumptions.

\subsection{What We Can Extract in Physics, If Equations (9)-(12) Hold?}

Simply put a relationship of the Lagrangian multiplier giving us the following:

$$
\lambda \sim \frac{1}{\kappa} \sqrt{\frac{-g}{\left(\delta g_{t t} \approx a_{\min }^{2} \phi\right)}} \cdot \Lambda .
$$

If the following is true, i.e. in a Pre Plankian to Planckian regime of spacetime

$$
\sqrt{\frac{-g}{\left(\delta g_{t t} \approx a_{\min }^{2} \phi\right)}} \approx \text { constant. }
$$

Then what has been done is to conflate the Lagrangian as equivalent to $\Lambda$ which if $\Lambda$ is also a constant is implying that the cosmological constant is obtaining for us the consomological constant value chosen as a precursor for (DE?) expansion of the universe, as given in the scale factors as of Equation (9) and Equation (8). i.e. what we are inferring then is similar to a result assumed by Padmanabhan, in [15].

\section{Conclusions}

But what is noticeable is that the inflaton equation as given by Padmanabhan [4] 
hopefully will not be incommensurate with the physics of the Corda Criteria given in the Gravity's breath document [16]. Keep in mind the importance of the result from reference [17] below which forms the core of Equation (15) below

$$
N_{e-\text { foldings }}=-\frac{8 \pi}{m_{\text {Planck }}^{2}} \cdot \int_{\phi 1}^{\phi 2} \frac{V(\phi)}{\left(\frac{\partial V(\phi)}{\partial \phi}\right)} \mathrm{d} \phi \geq 65 .
$$

Furthermore, we should keep in mind the physics incorporated in [18] [19], i.e. as to the work of LIGO. i.e. it is important to keep in mind that in addition, [20] has confirmed that a subsequent analysis of the event GW150914 by the LSC constrained the graviton Compton wavelength of those alternative theories of gravity in which the graviton is massive and placed a level of $90 \%$ confidence on the lower bound of $10^{13} \mathrm{~km}$ for a Compton wavelength of the graviton. Doing this sort of vetting protocols in line with being consistent with investigation as to a real investigation as to the fundamental nature of gravity. This is a way of confirming and showing via experimental data sets if general relativity is the final theory of gravitation. i.e., if massive gravity is confirmed, as given in [21], then GR is perhaps to be replaced by a scalar-tensor theory, as has been shown by Corda.

We can say though if we do confirm Equation (13) and Equation (14) that such observations may enable a more precise rendering of settling the issues brought up by references [16], and [21], as well as the appropriate use of the structures, algebraically given in [22] [23] for our comparison of the first integrals.

\section{Acknowledgements}

This work is supported in part by National Nature Science Foundation of China grant No. 11375279.

\section{References}

[1] Rovelli, C. and Vidotto, F. (2015) Covariant Loop Quantum Gravity. Cambridge University Press, Cambridge.

[2] Camara, C.S., de Garcia Maia, M.R., Carvalho, J.C. and Lima, J.A.S. (2004) Nonsingular FRW Cosmology and Non Linear Dynamics. arXiv:astro-ph/0402311.

[3] Ambjorn, J., Jurkiewicz, J., and Loll, R. (2010) Quantum Gravity as Sum over SpaceTimes. In: Boob-Bavnbek, B., Esposito, G. and Lesch, M., Eds., New Paths towards Quantum Gravity, Lecture Notes in Physics 807, Springer, Berlin, 59-124. https://doi.org/10.1007/978-3-642-11897-5_2

[4] Padmanabhan, T. (2005) Understanding Our Universe; Current Status, and Open Issues. In: Ashtekar, A., Ed., 100 Years of Relativity, Space-Time, Structure: Einstein and Beyond, World Scientific, Singapore, 175-204.

http://arxiv.org/abs/gr-qc/0503107 https://doi.org/10.1142/9789812700988_0007

[5] Penrose, R. (1965) Gravitational Collapse and Space-Time Singularities. Physical Review Letters, 14, 57-59. https://doi.org/10.1103/PhysRevLett.14.57

[6] Shankar, R. (1994) Principles of Quantum Mechanics. 2nd Edition, Springer, Berlin. 
https://doi.org/10.1007/978-1-4757-0576-8

[7] Roos, M. (2003) Introduction to Cosmology. 3rd Edition, Wiley Scientific, Hoboken.

[8] Karabulut, H. (2006) The Physical Meaning of Lagrange Multipliers. European Journal of Physics, 27, 709-718. https://arxiv.org/abs/0705.0609 https://doi.org/10.1088/0143-0807/27/4/003

[9] Spiegel, M. (1980) Theory and Problem of Theoretical Mechanics. Schaum's Outline Series, McGraw Hill, San Francisco.

[10] Landau, LD. and Lifshitz, E.M. (2005) Mechanics. 3rd Edition, in Course in Theoretical Physics, Vol. 1, Elsevier Books, Boston.

[11] Beckwith, A. (2016) Gedanken Experiment for Refining the Unruh Metric Tensor Uncertainty Principle via Schwarzschild Geometry and Planckian Space-Time with Initial Nonzero Entropy and Applying the Riemannian-Penrose Inequality and Initial Kinetic Energy for a Lower Bound to Graviton Mass (Massive Gravity). Journal of High Energy Physics, Gravitation and Cosmology, 2, 106-124.

https://doi.org/10.4236/jhepgc.2016.21012

[12] Giovannini, M. (2008) A Primer on the Physics of the Cosmic Microwave Background. World Press Scientific, Hackensack. https://doi.org/10.1142/6730

[13] Majumdar, D. (2016) Dark Matter: An Introduction. CRC Press, Boca Raton.

[14] Judson, T. (1994) Abstract Algebra, Theory and Applications. WS Publishing Company, Boston.

[15] Padmanabhan, T. https://www.physics.rutgers.edu/grad/690/Padmanabhan-2003.pdf

[16] Corda, C. (2012) Gravity's Primordial Breath. Electronic Journal of Theoretical Physics, 9, 1-10. http://www.ejtp.com/articles/ejtpv9i26p1.pdf

[17] Freese, K. (1992) Natural Inflation. In: Nath, P. and Recucroft, S., Eds., Particles, Strings, and Cosmology, World Scientific Publishing, Singapore, 408-428.

[18] Abbott, B.P., et al. (2016) Observation of Gravitational Waves from a Binary Black Hole Merger. Physical Review Letters, 116, Article ID: 061102. https://doi.org/10.1103/physrevlett.116.061102

[19] Abbott, B.P., et al. (2016) GW151226: Observation of Gravitational Waves from a 22-Solar-Mass Binary Black Hole Coalescence. Physical Review Letters, 116, Article ID: 241103. https://doi.org/10.1103/physrevlett.116.241103

[20] Abbott, B.P., et al. (2016) Tests of General Relativity with GW150914. https://arxiv.org/pdf/1602.03841.pdf

[21] Corda, C. (2009) Interferometric Detection of Gravitational Waves: The Definitive Test for General Relativity. International Journal of Modern Physics D, 18, 2275 2282. https://arxiv.org/abs/0905.2502 https://doi.org/10.1142/S0218271809015904

[22] Awodey, S. (2006) Category Theory. Oxford University Press, Oxford, 11. https://doi.org/10.1093/acprof:oso/9780198568612.001.0001

[23] Vinberg, Ė.B. (2003) A Course in Algebra. American Mathematical Society, 3. https://doi.org/10.1090/gsm/056 
Submit or recommend next manuscript to SCIRP and we will provide best service for you:

Accepting pre-submission inquiries through Email, Facebook, LinkedIn, Twitter, etc. A wide selection of journals (inclusive of 9 subjects, more than 200 journals)

Providing 24-hour high-quality service

User-friendly online submission system

Fair and swift peer-review system

Efficient typesetting and proofreading procedure

Display of the result of downloads and visits, as well as the number of cited articles Maximum dissemination of your research work

Submit your manuscript at: http://papersubmission.scirp.org/

Or contact jhepgc@scirp.org 